\title{
Préservation de la diversité génétique dans le genre Gossypium au Burkina Faso: collecte et évaluations préliminaires de cotonniers locaux
}

\author{
Larbouga BOURGOU ${ }^{1, *}$, Windpouiré Vianney TARPAGA ${ }^{2}$, Denys SANFO ${ }^{1}$, \\ Mahamadou SAWADOGO ${ }^{3}$ et Jean Didier ZONGO ${ }^{3}$ \\ ${ }^{1}$ Institut de l'Environnement et de Recherches Agricoles (INERA), \\ Programme Coton, 01 B.P: 208 Bobo-Dioulasso 01, Burkina Faso. \\ ${ }^{2}$ Institut de l'Environnement et de Recherches Agricoles (INERA), Programme Cultures Maraichères, \\ Fruitières et Plantes à Tubercules 01 B.P : 403 Bobo-Dioulasso 01, Burkina Faso. \\ ${ }^{3}$ Université de Ouagadougou, Laboratoire de Génétique et Biotechnologies Végétales, \\ 09 BP 848 Ouagadougou 09, Burkina Faso. \\ *Auteur correspondant; E-mail : diallabourgou@yahoo.fr; Tel : (00226)70135371
}

\section{RESUME}

Le but de l'étude a été de réunir et d'évaluer la diversité génétique des cotonniers traditionnels afin de les valoriser et de les conserver. Dans 243 villages, 336 accessions ont été collectées. Pendant la campagne hivernale 2010-2011, une évaluation préliminaire a été faite à partir de 25 caractères qualitatifs et quantitatifs. Les résultats ont révélé la présence d'un grand nombre d'écotypes différents appartenant aux espèces diploïdes (Gossypium arboreum et $G$. herbaceum) et tétraploïdes ( $G$. hirsutum et $G$. barbadense) de cotonniers cultivés. Au plan qualitatif, les caractères «forme des feuilles», «forme des capsules» et «couleur du duvet» ont été les plus discriminants, subdivisant les accessions en 3 groupes. Les variables quantitatives, ont montré une opposition nette entre celles liées à l'architecture et celles liées à la production. L'ACP a mis en exergue 6 groupes de diversité. Les groupes G1, G2 et G3 étaient homogènes. Les autres groupes, cosmopolites, et la forte dispersion d'accessions dans le plan confirment l'importante diversité génétique intra- et inter-écotypes. Cette richesse génétique sera conservée, surtout son évaluation approfondie est en cours. Les cotonniers locaux jadis négligés pourraient s'avérer utiles dans un contexte de changements climatiques et de rétrécissement continu de la base génétique des variétés cultivées.

(C) 2014 International Formulae Group. All rights reserved.

Mots clés : Burkina Faso, cotonniers traditionnels, Gossypium spp., prospection-collecte, diversité morphologique.

\section{INTRODUCTION}

Jusqu'au début du 19 siècle, la culture $\mathrm{du}$ coton restait l'apanage de l'Orient, principalement de l'Inde, et aussi des parties tropicales de l'Afrique pour satisfaire les besoins d'une industrie manuelle de modeste importance ou de l'artisanat locale (Levrat, 2008). Puis, elle a évolué dans le temps, 
passant d'une culture traditionnelle à une culture industrielle, source de stabilité économique et d'investissement de développement au sens plus large (Zoundi et al., 2006). Pour les pays producteurs de 1'Afrique de l'Ouest dont le Burkina Faso, la culture du coton a constitué l'un des rares succès après les indépendances (Lagandre, 2005). Ces dernières années, la stagnation voire la baisse des rendements apparaît préoccupante. Les raisons sont multiples (dégradation des sols, pressions parasitaires, changements climatiques, etc.) et dénotent également d'une faible amélioration des cotonniers cultivés due à une utilisation insuffisante de la diversité génétique au sein du genre. Le pool génétique constitué uniquement de $G$. hirsutum est de plus en plus appauvri puisqu'il n'a plus été alimenté depuis l'arrêt des activités de l'IRCT (Institut de Recherches du Coton et des Textiles exotiques) en Afrique (Dessauw et Hau, 2006 ; N’Doye et al., 2011). Des études sur les croisements interspécifiques, les lignées monosomiques d'addition et les biotechnologies modernes ont montré les possibilités d'utiliser une large gamme de ressources génétiques pour élargir la base génétique trop étroite du cotonnier cultivé (Hau, 2000 ; Mergeai, 2006 ; Sarr et Mergeai, 2009).

Plus récemment, quand on a constaté une érosion de ces ressources génétiques et une uniformisation génétique des variétés cultivées, la notion de collecte et de conservation est plus que d'actualité au plan international (Wallace et al., 2009). De nombreuses collectes ont eu lieu en Amérique latine depuis les années 1980 et se poursuivent encore (Ulloa et al., 2006). L'Australie, la Chine et l'Inde possèdent des collections de plus en plus remarquables et de plus en plus caractérisées (Singh et al., 2003 ; Gotemare et singh, 2004). L'Ouzbékistan (plus de 10000 entrées) suivi des USA et de l'Inde (plus de 9000 entrées), de la Chine (plus de 7000 entrées), de la Russie (plus de
6000 entées) et du Cirad (plus de 4000 entrées) possèdent les plus importantes collections mondiales de cotonniers (FAO, 2010). En revanche, l'Afrique qui occupe une place importante dans la production mondiale du coton est restée une zone peu explorée (Dessauw et Hau, 2006). En outre, l'industrialisation de la culture du coton s'est traduite par une éradication des variétés traditionnelles au profit des variétés introduites. Les efforts de collecte au Sénégal (Bèye, 1989) et au Cameroun (Seignobos et Schwendiman, 1991) n'ont pas été suivis de caractérisations poussées ni au champ ni sur le plan moléculaire et ceci a limité les possibilités de valorisation/conservation de leurs collections.

$\mathrm{Au}$ Burkina Faso et à notre connaissance, aucun document ne fait état de collecte de cotonniers à des fins de conservation et de valorisation. Néanmoins, on retrouve quelques pieds, en situation très relictuelle, dans des concessions, des jardins et rarement dans des champs. Ils ont été conservés pour des usages divers tels la pharmacopée, les rituels coutumiers, etc. (Père, 2004). Les objectifs de la présente étude étaient de constituer une collection d'écotypes de cotonniers à l'échelle du Burkina Faso pour des besoins de recherche et de procéder à une description sommaire de cette collection sur le plan agromorphologique.

\section{MATERIEL ET METHODES}

\section{Zones et sites de collecte}

La prospection-collecte s'est déroulée sur l'étendue du Burkina Faso comprise entre $9^{\circ} 20^{\prime}$ et $15^{\circ} 3^{\prime}$ de Latitude Nord, $2^{\circ} 20^{\prime}$ de Longitude Est et $5^{\circ} 3^{\prime}$ de Longitude Ouest. Le climat y est tropical, subdivisé en trois zones climatiques de gradient d'humidité nord-sud. La végétation est stratifiée à l'image des zones climatiques sur un relief plat à $75 \%$. Les sols, de fertilité relativement faible notamment en phosphore et en azote, sont classés en neuf types avec une dominance des 
sols à sesquioxydes de fer et de manganèse (Bado, 2002). Au Burkina Faso, vivent une soixantaine d'ethnies qui ont eu et qui continuent d'avoir des liens coutumiers divers avec le cotonnier et ses produits. De nos jours, la carte du coton divise le pays en une zone cotonnière de production industrielle (> $600 \mathrm{~mm} / \mathrm{an}$ ) et une zone hors coton $(\leq 600 \mathrm{~mm} / \mathrm{an})$ où la production industrielle est impossible mais où on note la présence relictuelle de cotonniers surtout pluriannuels. Plus de 500 localités ont été visitées mais seuls les villages administratifs où a été collecté au moins un échantillon ont été illustrés (Figure 1).

\section{Modalités de réalisation de la collecte}

Comme en Inde (Singh et al., 2003), le maximum de villages a été visité afin de collecter le maximum d'accessions et de générer le plus d'informations sur la diversité génétique étudiée. L'opération a été conduite entre novembre 2008 et mai 2010. Dans chaque localité, des personnes-ressources (responsables coutumiers, agents d'agriculture à la retraite) ont été approchées. Les informations recueillies nous ont orientés vers les détenteurs du matériel végétal recherché.

Un questionnaire de collecte comportant l'identité du détenteur, l'origine du matériel, les usages qui en sont faits et les contraintes dans la valorisation a été rempli à chaque prélèvement. Dans les zones cotonnières, les écotypes locaux ont été distingués des cotonniers en culture industrielle par des caractéristiques morphologiques précises : port, feuilles, cycle, taille, etc. Le matériel à collecter se composait de capsules ou de coton-graine récoltés sur pieds et/ou des graines reçues en petite quantité de certains détenteurs (Ibpgr, 1985). Afin d'illustrer la diversité morphologique, des clichés de plantes et/ou d'organes (port du plant, capsules, feuilles, fleurs, etc.) ont été réalisés.

\section{Site expérimental}

L'évaluation au champ de la collection a été conduite à la station de Farako-Bâ située dans la zone cotonnière ouest et comprise entre les isohyètes 800 et 1200 mm. Les coordonnées géographiques du site sont : $4^{\circ} 20^{\prime}$ longitude Ouest, $11^{\circ} 06^{\prime}$ latitude Nord et $405 \mathrm{~m}$ d'altitude. Farako-Bâ a un climat sud-soudanien, une végétation de type savane arborée à arbustive. Les sols y sont de type ferrugineux tropicaux, pauvres en argile et en matière organique. Ils sont de texture sablolimoneuse, légèrement acides et pauvres en azote et phosphore (Bado, 2002).

\section{Dispositif expérimental}

Etant donné le caractère préliminaire de l'évaluation et en raison du grand nombre d'accessions avec probablement des doublons pour certaines d'entre elles, nous avons opté pour un dispositif sans répétition. Les accessions ont été aléatoirement distribuées dans les parcelles élémentaires. Chaque parcelle élémentaire était constituée de 3 lignes de $10 \mathrm{~m}$ pour chaque accession. Les semis ont été réalisés aux écartements de 0,8 m x 0,8 m. Au total, 308 accessions plus la variété FK37 insérée comme témoin (en 13 entrées) ont été semées le 25 juin 2010. La fertilisation a consisté en un apport de NPKSB (14-18-18-6-1) à $150 \mathrm{~kg} / \mathrm{ha}$ et d'Urée $(46 \% \mathrm{~N})$ à $50 \mathrm{~kg} / \mathrm{ha}$, respectivement, 20 et 45 jours après semis (jas). La protection phytosanitaire a été réalisée avec les produits suivants: Avaunt 150 EC (Indoxacarb 150 g/L) suivi du Lambdacal P 636 EC (Zétaméthrine $36 \mathrm{~g} / \mathrm{L}$ + profenofos $600 \mathrm{~g} / \mathrm{L}$ ) puis du Conquest C176 EC (Cyperméthrine $144 \mathrm{~g} / \mathrm{L}+$ acétamipride $32 \mathrm{~g} / \mathrm{L}$ ), utilisés en 6 traitements espacés de 2 semaines. Les traitements ont débuté 30 jas aux doses respectives 0,$17 ; 0,334$ et 0,25 litre/ha pour chaque insecticide.

\section{Collecte des données}

Des caractères qualitatifs et quantitatifs suffisamment discriminants et 
couramment utilisés pour décrire les cultivars et les cultigroupes de cotonniers ont été retenus sur 5 plantes de chaque ligne centrale (Ibpgr, 1985 ; Upov, 2001).

Les données qualitatives ont été appréciées, de façon visuelle en cours de végétation, à partir des feuilles, des fleurs, de la plante, des capsules et des graines. Chaque caractère observé comportait plusieurs modalités comme décrites au Tableau 1. Les variables quantitatives étaient liées d'une part aux branches végétatives (BV) et d'autre part aux branches fructifères (BF). Celles liées aux BV ont trait à l'architecture de la plante tandis celles liées aux BF renseignaient sur la productivité et la production. Il s'agissait du nombre de branches végétatives (NBV) et fructifères $(\mathrm{NBF})$, du nombre de capsules produites sur les branches végétatives (CBV) et fructifères $(\mathrm{CBF})$, le nombre de sites des branches végétatives (SBV) et fructifères (SBF), le nombre de nœuds sur la tige principale (NN) et la taille de la plante (HT). Elles ont été mesurées à l'issue d'un «plant mapping » (Sékloka, 2006) en décembre 2010.

\section{Analyse des données}

Les données ont été triées sur Excel afin d'écarter toutes les accessions qui présentaient moins de 5 individus. Ainsi, 90 échantillons ont été écartés des analyses statistiques. Par ailleurs, 31 échantillons semés n'ont présenté aucun plant viable. Finalement, 200 accessions ont fait l'objet d'analyses statistiques. En ce qui concerne les caractères qualitatifs, nous avons calculé les fréquences $(\%)$ des différentes modalités observées pour chaque caractère. Pour les accessions qui présentaient plus d'une modalité pour un caractère donné, une modalité supplémentaire nommée « hétérogène » a été définie. Les données quantitatives ont subi une analyse descriptive et une analyse en composantes principales (ACP) avec le logiciel StatBox Agri. Ces analyses visaient à décrire la diversité et à révéler l'organisation en groupes d'accessions semblables ou dissemblables.

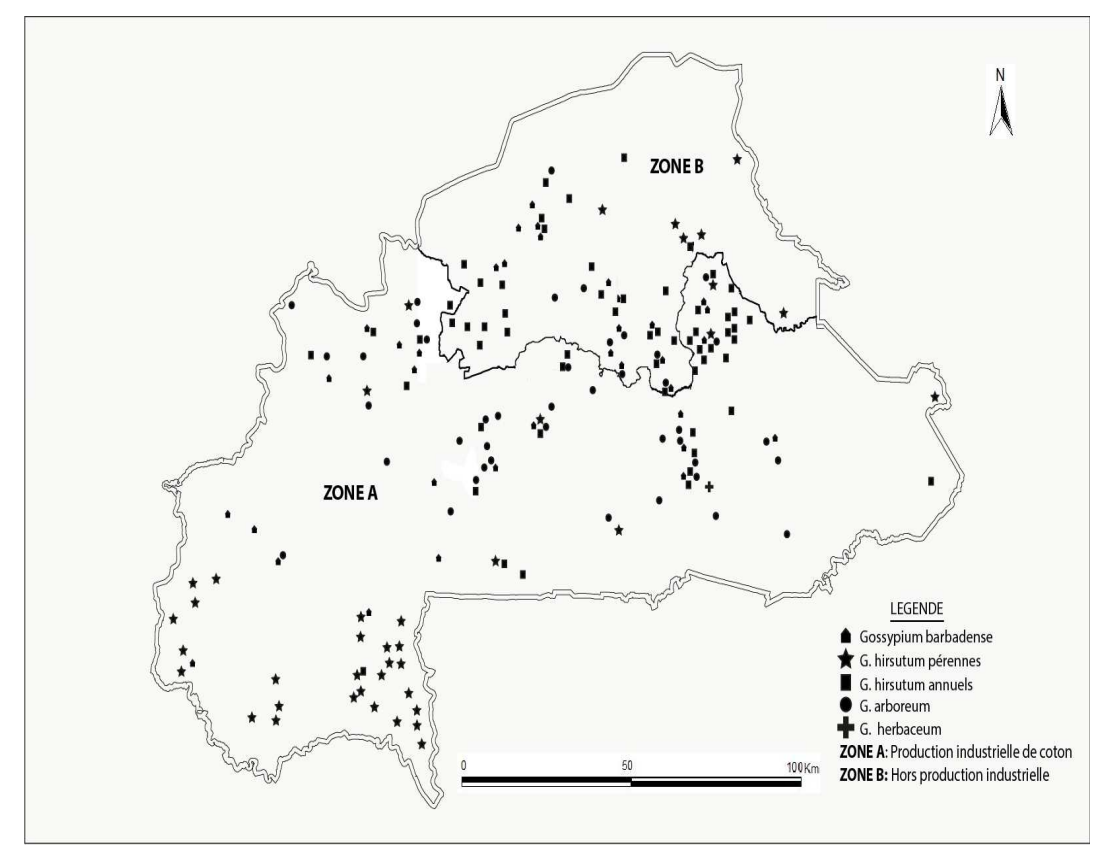

Figure 1 : Sites de collecte et types d'accessions collectées. 


\section{RESULTATS}

Analyse descriptive des variables morphologiques quantitatives

Le Tableau 1 montre qu'à la date où les variables ont été mesurées, certaines accessions ont présenté des valeurs minimales nulles pour chacune des variables sauf pour NN et HT. En outre, de grands écarts existent entre les accessions puisque certaines présentaient des valeurs nulles pour une variable donnée, tandis que d'autres ont donné des valeurs très élevées pour la même variable comme le montrent les maxima. Toutes ces remarques sont corroborées par les CV très élevés, surtout pour les variables en rapport avec les branches végétatives $(\mathrm{NBV}=150,97 \% ; \mathrm{CBV}=180,40 \%$ et $\mathrm{SBV}$ $=130,94 \%$ ).

\section{Observation des caractères qualitatifs}

L'observation de caractères qualitatifs met en exergue différents types morphologiques à l'intérieur de chaque caractère (Tableau 2). Pour l'ensemble des caractères, une modalité a toujours tendance à la prédominance. Ce constat est net sur les caractères «forme des feuilles » $(82,5 \%$ pour la forme palmée), « expression de la macule rouge » (absente chez 90\% des individus), « forme des capsules » (arrondie pour $82 \%$ des individus), « attache des graines » (graines libres à 92\%) et « couleur de la fibre » (blanche à 98\%). A l'opposé, certaines caractéristiques sont plutôt rares comme la couleur blanche des pétales et la couleur brune de la fibre $(1,5 \%)$. Il existe également des caractères à fréquence inférieure à $1 \%$ comme la forte pilosité des feuilles, la grande précocité, la couleur kaki de la fibre. Mais pour la plupart des caractères en l'occurrence la taille des feuilles, la pilosité des feuilles, la couleur des pétales, la taille des capsules, l'époque de déhiscence des capsules et la taille des graines, la prédominance d'un type morphologique n'empêche pas la présence notable d'autres variantes du même caractère.

\section{Regroupement des écotypes par ACP}

Les deux premières composantes principales ( $\mathrm{CP} 1$ et $\mathrm{CP} 2)$ ont été retenues pour décrire la diversité totale de la collection. Elles expliquent plus de $83 \%$ de cette diversité (Tableau 3). L'axe 1 (CP1) renferme $52,30 \%$ de la variation et fait ressortir des corrélations intéressantes et fortes entre NBV et HT d'une part $(78,30 \%)$ et entre HT et NN d'autre part $(79,00 \%)$. $\mathrm{NBV}$, de part sa forte contribution à la constitution de l'axe $(22,47 \%)$ est la variable expliquant le mieux CP1 suivie de SBV $(21,23 \%)$, NN $(17,73 \%)$ et HT $(15,69 \%)$

(Tableau 3). Ces variables sont caractéristiques du développement végétatif, de la vigueur générale de la plante ce qui permet d'associer à cet axe le caractère primitif et l'architecture des écotypes. Le deuxième axe décrit $30,78 \%$ de la variabilité. Dans l'ordre d'importance de leurs contributions et de leur plus ou moins bonne représentation dans le plan, l'axe 2 associe SBF $(31,54 \%), \quad \mathrm{CBF}(26,07 \%)$ et $\mathrm{NBF}$ $(25,35 \%)$. A travers cet axe, c'est la productivité et la production réelle des écotypes qui sont expliquées puisque, chez le cotonnier, l'essentiel de la production provient de ces branches.

La représentation des écotypes dans le plan défini par ces deux axes montre une dispersion plus ou moins structurée des écotypes. Un diagnostic attentif, et en se référant aux caractéristiques morphologiques analysées plus haut, permet d'isoler 6 groupes d'écotypes au regard de la représentation du plan 1/2 de l'ACP (Figure 2). Les groupes G1, G2 et G3 sont nommés suivant des informations issues de l'analyse des variables qualitatives. En dépit de la diversité des origines, des écotypes très homogènes ayant des caractéristiques 
qualitatives très proches, constituent $\mathrm{G} 2$ et G3. Par rapport aux paramètres (NBV, SBV, NN) définissant l'axe 1, les écotypes du G3 ont des valeurs proches de la moyenne de la collection tandis que ceux du G2 présentent des valeurs bien supérieures. Par rapport à 1'axe 2, G2 et G3 se ressemblent; leurs individus sont de valeurs très faibles pour le nombre de BF, de nœuds par BF et de capsules des BF. La Figure 2 montre que G1 est étiré, avec des individus préférentiellement corrélés à $\mathrm{CP} 2$, de valeurs allant de moyenne à bonne pour cet axe. Par rapport à $\mathrm{CP} 1$ par contre, ces individus sont homogènes, à valeurs en NBV et en CBV très faibles à nulles. Les accessions de ce groupe, y compris toutes les entrées du témoin, paraissent courts et précoces.

Les groupes G4, G5 et G6 ont été définis en raison de leurs particularités vis-àvis des groupes précédents (Figure 2). Leurs individus sont dissemblables au regard des caractéristiques qualitatives mais semblables du point de vue des caractères mesurés. G4 comprend les écotypes les plus corrélés à l'axe 1, de caractéristiques qualitatives semblables à celles de G2 d'une part et G3 d'autre part. Les accessions du groupe 4 font partie des cotonniers les plus hauts avec une taille moyenne de plus de $200 \mathrm{~cm}$ et un mode de croissance indéterminé. En outre, E298, E212, E297 produisent des BV et des SBV en très grands nombres qui font qu'ils sont morphologiquement à l'opposé des écotypes de G5 pour ces caractères. Les individus du G5 sont ceux qui produisent le plus de BF et retiennent le plus de capsules sur leurs BF. Ils sont assez précoces, de taille moyenne avec très peu de BV. Si la plupart des individus de ce groupe ont des caractéristiques phénotypiques semblables à celles de G1, E275 est une exception puisque ses caractéristiques phénotypiques sont celles de G2. Enfin, le groupe 6 (G6) est préférentiellement corrélé à l'axe 1 , avec des individus de valeurs assez moyennes pour les paramètres de l'axe 2. Ils sont semblables à la fois à ceux des G1, G2 et G3.

Tableau 1 : Analyse descriptive des variables mesurées.

\begin{tabular}{lccccccc}
\hline Caractères & Ind. & Min & Médiane & Max & Moy. & E.T & C.V (\%) \\
\hline NBV & 1000 & 0,00 & 2,00 & 45,00 & 4,43 & 6,729 & 151,97 \\
CBV & 1000 & 0,00 & 1,00 & 105,00 & 3,28 & 5,912 & 180,40 \\
SBV & 1000 & 0,00 & 14,00 & 310,00 & 30,30 & 39,676 & 130,94 \\
NBF & 1000 & 0,00 & 15,00 & 83,00 & 15,06 & 5,652 & 37,52 \\
CBF & 1000 & 0,00 & 6,00 & 59,00 & 8,31 & 5,645 & 67,96 \\
SBF & 1000 & 0,00 & 29,00 & 106,00 & 31,82 & 16,633 & 52,27 \\
NN & 1000 & 3,00 & 29,00 & 110,00 & 30,76 & 7,006 & 22,77 \\
HT & 1000 & 35,00 & 97,75 & 448,00 & 103,66 & 36,380 & 35,09 \\
\hline
\end{tabular}


L. BOURGOU et al. / Int. J. Biol. Chem. Sci. 8(5): 2081-2094, 2014

Tableau 1 : Modalités des caractères observés et leurs fréquences.

\begin{tabular}{|c|c|c|c|c|c|c|c|}
\hline $\begin{array}{l}\text { Partie observée } \\
\text { de la plante }\end{array}$ & $\begin{array}{l}\text { Caractères } \\
\text { analysés }\end{array}$ & $\begin{array}{l}\text { Modalités } \\
\text { (variables) }\end{array}$ & $\begin{array}{c}\text { Fréquence } \\
(\%)\end{array}$ & $\begin{array}{c}\text { Partie observée } \\
\text { de la plante }\end{array}$ & $\begin{array}{l}\text { Caractères } \\
\text { analysés }\end{array}$ & $\begin{array}{l}\text { Modalités } \\
\text { (variables) }\end{array}$ & $\begin{array}{c}\text { Fréquence } \\
(\%)\end{array}$ \\
\hline \multirow{13}{*}{ Feuille } & \multirow{3}{*}{$\begin{array}{l}\text { Forme } \\
\text { (FFE) }\end{array}$} & Palmée & 82,5 & \multirow{13}{*}{ Plante } & \multirow{5}{*}{$\begin{array}{l}\text { Taille } \\
\text { (TPL) }\end{array}$} & Petite & 11,0 \\
\hline & & Palmée à digitée & 8,5 & & & Moyenne & 52,5 \\
\hline & & Digitée & 9,0 & & & Grande & 8,50 \\
\hline & \multirow{4}{*}{$\begin{array}{l}\text { Taille } \\
\text { (TFE) }\end{array}$} & Petite & 22,0 & & & Très grande & 6,5 \\
\hline & & Moyenne & 68,5 & & & Hétérogène & 21,5 \\
\hline & & Grande & 9,0 & & \multirow{4}{*}{$\begin{array}{c}\text { Port de la } \\
\text { plante } \\
\text { (PPL) }\end{array}$} & Cluster & 11,0 \\
\hline & & Hétérogène & 0,5 & & & Gobelet & 62,5 \\
\hline & $\begin{array}{l}\text { Pilosité de } \\
\text { la face }\end{array}$ & Nulle/très faible & 24,0 & & & Conique & 4,0 \\
\hline & \multirow{5}{*}{$\begin{array}{l}\text { inférieure } \\
\text { (PFE) }\end{array}$} & Faible & 71,5 & & & Hétérogène & 22,5 \\
\hline & & Moyenne & 3,5 & & \multirow{4}{*}{$\begin{array}{l}\text { Densité du } \\
\text { feuillage } \\
\text { (FPL) }\end{array}$} & Lâche & 8,5 \\
\hline & & Eort & 05 & & & Moyenne & 75,5 \\
\hline & & 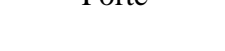 & , & & & Dense & 14,0 \\
\hline & & Hétérogène & 0,5 & & & Hétérogène & 2,0 \\
\hline \multirow{4}{*}{ Capsule } & \multirow{4}{*}{$\begin{array}{l}\text { Taille } \\
\text { (TCA) }\end{array}$} & Petite & 36,0 & \multirow{4}{*}{ Fleur } & \multirow{4}{*}{$\begin{array}{c}\text { Couleur des } \\
\text { pétales }\end{array}$} & Crème & 68,0 \\
\hline & & Moyenne & 60,5 & & & Jaune & 22,0 \\
\hline & & Grosse & 3,0 & & & Rouge & 7,5 \\
\hline & & Hétérogène & 0,5 & & & Blanche & 1,5 \\
\hline
\end{tabular}


L. BOURGOU et al. / Int. J. Biol. Chem. Sci. 8(5): 2081-2094, 2014

\begin{tabular}{|c|c|c|c|c|c|c|c|}
\hline & Forme de la & Arrondie & 82,5 & & & \multirow{2}{*}{ Hétérogène } & \multirow{2}{*}{1,0} \\
\hline & capsule & Conique & 8,5 & & & & \\
\hline & (FCA) & Pyramidale & 9,0 & & \multirow[b]{2}{*}{ Couleur du } & \multirow{2}{*}{ Crème } & \multirow{2}{*}{76,5} \\
\hline & \multirow{6}{*}{$\begin{array}{c}\text { Epoque de } \\
\text { déhiscence } \\
\text { (DCA) }\end{array}$} & Très précoce & 0,5 & & & & \\
\hline & & Précoce & 59,5 & & pollen & Jaune & 19,5 \\
\hline & & Moyenne & 11,0 & & \multirow[t]{2}{*}{ (CPO) } & \multirow[t]{2}{*}{ Jaune foncé } & \multirow{2}{*}{4,0} \\
\hline & & Tardive & 20,5 & & & & \\
\hline & & Très tardive & 8,0 & & \multirow{2}{*}{$\begin{array}{l}\text { Macule } \\
\text { rouge } \\
\text { (MRO) }\end{array}$} & Présence & 10,0 \\
\hline & & Hétérogène & 0,5 & & & Absence & 90,0 \\
\hline \multirow{9}{*}{ Semences } & Attache des & Soudées & 80 & \multirow{9}{*}{ Semences } & \multirow{3}{*}{$\begin{array}{l}\text { Duvet } \\
\text { (DUV) }\end{array}$} & Présence & 89,0 \\
\hline & graines & & & & & Absence & 9,5 \\
\hline & (AGR) & Libres & 92,0 & & & Hétérogène & 1,5 \\
\hline & Taille des & Petites & 41,5 & & \multirow{6}{*}{$\begin{array}{l}\text { Couleur du } \\
\text { duvet } \\
\text { (CDU) }\end{array}$} & Blanche & 74,0 \\
\hline & graines & Moyennes & 53,5 & & & Verte & 10,0 \\
\hline & (TGR) & Grosses & 5,0 & & & Brune & 4,0 \\
\hline & Couleur de 1 & Blanche & 98,0 & & & Kaki & 0,5 \\
\hline & a fibre & Brune & 1,5 & & & Absence & 9,0 \\
\hline & $(\mathrm{CFI})$ & Kaki & 0,5 & & & Hétérogène & 2,5 \\
\hline
\end{tabular}


Tableau 3 : Résumé de l'ACP : Cosinus carrés, valeurs et contributions des variables aux axes de l'ACP.

\begin{tabular}{|c|c|c|c|c|c|c|}
\hline \multirow[b]{2}{*}{ Variables } & \multicolumn{2}{|r|}{ F1 } & \multicolumn{2}{|r|}{$\mathbf{F} 2$} & \multicolumn{2}{|r|}{ F3 } \\
\hline & $\begin{array}{l}\text { Cosinus } \\
\text { carrés }\end{array}$ & $\begin{array}{c}\text { Contribution } \\
(\%)\end{array}$ & $\begin{array}{l}\text { Cosinus } \\
\text { carré }\end{array}$ & $\begin{array}{c}\text { Contribution } \\
(\%)\end{array}$ & $\begin{array}{c}\text { Cosinus } \\
\text { carré }\end{array}$ & $\begin{array}{c}\text { Contribution } \\
(\%)\end{array}$ \\
\hline NBV & 0,940 & 22,469 & 0,001 & 0,026 & 0,000 & 0,006 \\
\hline CBV & 0,525 & 12,559 & 0,176 & 7,139 & 0,173 & 30,197 \\
\hline SBV & 0,888 & 21,227 & 0,024 & 0,970 & 0,007 & 1,180 \\
\hline $\mathrm{NBF}$ & 0,196 & 4,681 & 0,624 & 25,349 & 0,084 & 14,658 \\
\hline $\mathrm{CBF}$ & 0,086 & 2,062 & 0,642 & 26,070 & 0,153 & 26,608 \\
\hline SBF & 0,150 & 3,584 & 0,777 & 31,538 & 0,018 & 3,170 \\
\hline $\mathrm{NN}$ & 0,742 & 17,733 & 0,030 & 1,204 & 0,083 & 14,458 \\
\hline HT & 0,656 & 15,686 & 0,190 & 7,704 & 0,056 & 9,723 \\
\hline $\begin{array}{l}\text { Valeurs } \\
\text { propres }\end{array}$ & \multicolumn{2}{|r|}{4,184} & \multicolumn{2}{|r|}{2,462} & \multicolumn{2}{|r|}{0,573} \\
\hline $\begin{array}{l}\text { Variance } \\
(\%)\end{array}$ & \multicolumn{2}{|c|}{52,301} & \multicolumn{2}{|r|}{30,779} & \multicolumn{2}{|r|}{7,167} \\
\hline
\end{tabular}

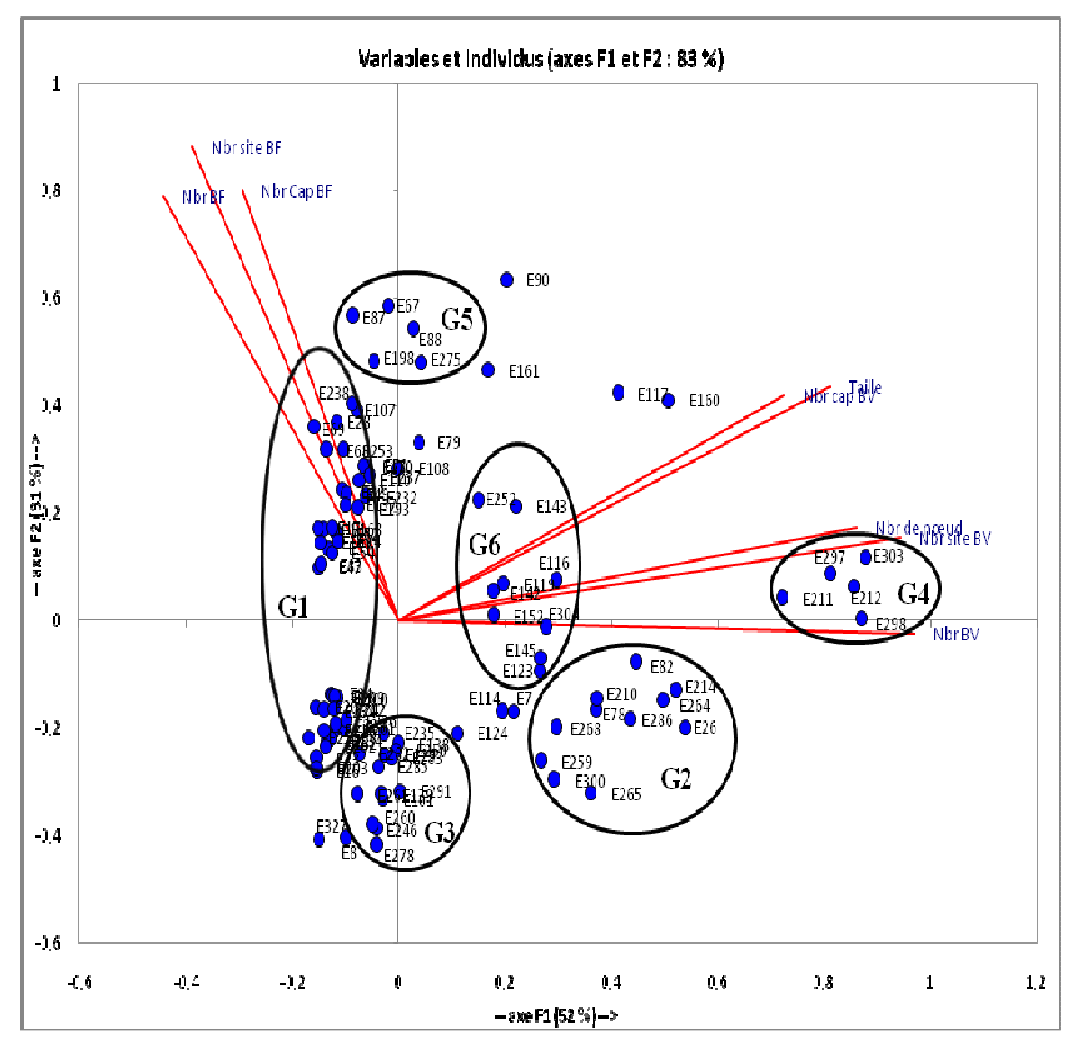

Figure 2 : Structuration des accessions dans le plan défini par les axes 1 et 2 de l'ACP. 


\section{DISCUSSION}

Etat des lieux sur les cotonniers traditionnels au Burkina Faso

Le dépouillement des fiches d'enquête indique la présence de cultivars locaux de cotonniers dans plus de 240 villages répartis sur l'ensemble des zones climatiques du Burkina Faso. Dans la majorité des cas, il s'agit de très vieux plants, rarement au-delà de trois pieds, isolés dans les concessions ou dans les jardins. Dans de très rares cas, des champs ont été rencontrés avec des superficies toujours inférieures à 0,25 ha. Aucun écotype n'a pu être collecté à l'état sauvage. Au total, 358 échantillons ont été réunis comprenant des cotonniers traditionnels ou indigènes si l'on se réfère à la définition consacrée à ces derniers par Seignobos et Schwendiman (1991) et Bèye (1989). Pour des raisons identitaires et des besoins de rituels divers (Père, 2004), les populations rurales ont joué un rôle important dans la conservation et la gestion des ressources génétiques des cotonniers traditionnels. Ce rôle est d'autant plus important que les nombreuses tentatives de valorisation de celles-ci se sont soldées par des échecs, tandis que les interdictions de leur culture à certaines époques au profit des variétés améliorées les prédisposaient à une disparition inéluctable (Seignobos et Schwendiman, 1991 ; Levrat, 2008). La majeure partie des populations déclare qu'il y a eu des introductions de cotonniers (exemple de la Mecque). Cependant, chaque groupe ethnique s'approprie l'origine de ses écotypes à travers l'attribution d'un nom vernaculaire tels que «Sana kwoara » pour désigner le cotonnier des San, «Mooghonabalaamdo » pour celui de l'empereur des Mossi, « Gourounkounkoundou » pour le cotonnier des Gourmantché, etc.. Ils distinguent ainsi leurs écotypes des cotonniers améliorés par le cycle (pluriannuel) et la rusticité. Aussi, leurs opinions convergent à expliquer la situation relictuelle actuelle des cotonniers traditionnels comme le résultat de la concurrence des cotonniers améliorés (plus productifs), le recul de l'artisanat local face à l'essor des tissus importés et de la friperie, ainsi que du manque d'intérêt réel pour une promotion de ces cotonniers (Igué, 2003). Selon eux, la dégradation climatique n'apparâit que comme contrainte de second ordre étant donnée la forte rusticité des cotonniers indigènes.

\section{Morphologie et génétique des cotonniers collectés}

Analyse des caractères qualitatifs

En se référant à des descriptions morphologiques et taxonomiques antérieures dans le genre Gossypium (Valicek, 1978 ; Fryxell, 1992 ; Dessauw et Hau, 2004 ; Bednarz et Nichols, 2005), notre collection est riche d'au moins quatre espèces : deux diploïdes ( $G$. arboreum et $G$. herbaceum) et deux tétraploïdes ( $G$. hirsutum et $G$. barbadense). Les caractères qualitatifs analysés démontrent une grande diversité d'un écotype à l'autre et aussi une variabilité intra-écotype.

Trois groupes se dégagent nettement sur la base des fréquences des modalités des différents caractères. Le groupe 1 est constitué d'écotypes dont les individus dominent dans la population vu que l'expression de leurs caractéristiques constitue la fréquence la plus élevée. Ce groupe est caractérisé par les plantes au port en gobelet, de taille moyenne avec des feuilles palmées de taille moyenne, des fleurs à pétales et pollen de couleur crèmes sans macule rouge et des capsules de forme arrondie s'ouvrant précocement pour donner des graines libres, à fibre et duvet blancs. Le témoin FK37 se retrouve dans ce groupe ; ces écotypes sont donc identifiés comme des variétés de $G$. hirsutum exploitées par les sociétés cotonnières dans un passé plus ou 
moins récent. Les groupes 2 et 3 sont constitués d'accessions dont les caractéristiques les plus représentatives sont sensiblement égales en valeurs. Ces deux groupes se distinguent au niveau de 3 caractères : FFE avec le groupe 2 à feuilles digitées (9\% de l'effectif) et le groupe 3 à feuilles palmées-à-digitées $\quad(8,5 \%$ des individus) ; FCA avec le type pyramidal pour les individus du groupe $2(9 \%)$ et le type conique pour les écotypes du groupe 3 $(8,5 \%)$; CDU, avec la modalité «verte » exprimée par les individus du groupe $2(10 \%)$ et « absence de duvet » exprimée par les individus du groupe $3(9 \%)$. Pour le caractère FPL, nous avons le type «lâche » qui concerne les individus du groupe $2(8,5 \%)$ tandis que le type «dense » concerne les écotypes groupe 3 (14\%). Pour ce qui concerne le caractère CPE, 7,5\% des écotypes de la collection expriment des pétales de couleur rouge (groupe 2) alors que $22 \%$ ont des pétales jaunes, incluant des individus du groupe 3 . Au regard de l'identification des espèces, le groupe 2 décrit une variabilité de $G$. arboreum (et $G$. herbaceum) tandis que le groupe 3 décrit la variabilité de G. barbadense.

Des quatre espèces qui constituent notre collection, $G$. hirsutum et $G$. barbadense (tétraploïdes) sont originaires d'Amérique du Sud et introduites en Afrique de l'Ouest dès le 16e siècle dans le cadre du commerce triangulaire (Bèye, 1989; Hau, 2000). Quant aux espèces diploïdes $G$. arboreum et $G$. herbaceum, elles ont sans doute été introduites en provenance d'Asie ou des parties orientales de l'Afrique depuis une époque beaucoup plus ancienne (Hau, 2000 ; Levrat, 2008). Des analyses plus poussées au champ et sur le plan moléculaire sont en cours pour parvenir à une caractérisation plus fine afin de permettre une classification plus approfondie.

\section{Analyse du regroupement des écotypes par $A C P$}

L'ACP des écotypes met en exergue leur diversité et leur organisation en six (06) groupes plus ou moins spécifiques. A l'image de FK37, le G1 majoritaire, est constitué de cotonniers de G. hirsutum qui ont été plus ou moins améliorées pour une culture industrielle à cycle annuelle. En outre, nombre d'individus hors groupes, très dispersés, appartiennent à G. hirsutum; ce qui traduit une fois de plus la plasticité de cette espèce et sa plus forte valorisation en cotonculture (sêkloka, 2006). Les groupes G2 et G3 regroupent des écotypes à forte tendance végétative et qui ont eu tendance à peu produire en cette première année de plantation. Ces résultats sont similaires à ceux de Bèye (1989) et Seignobos et Schwendiman (1991) qui ont rapporté que les cotonniers indigènes ( $G$. arboreum, $G$. herbaceum) avec $G$. barbadense produisent très faiblement en première année de plantation du fait qu'ils n'ont pas été assez améliorés ou du système de culture insuffisamment propice. Par ailleurs, en bonnes conditions environnementales, ces types morphologiques sont pérennes et exceptionnellement grands ; ce qui conférèrent une tendance lianescente à $G$. arboreum en cas de proximité de support (Valicek, 1978 ; Seignobos et Schwendiman, 1991). La présence dans G6 d'écotypes proches de ceux de G1 (plus de 15) et bien corrélés à l'axe 1 est remarquable. Il pourrait s'agir de cotonniers sub-spontannés ou « échappés de culture » des anciennes introductions de G. hirsutum. Au regard de leurs caractéristiques, ces écotypes pourraient n'avoir jamais fait l'objet d'exploitation industrielle par les sociétés cotonnières actuelles. Ils semblent cependant bien adaptés aux conditions du milieu ouest africain par leur plasticité génétique (Bèye, 1989). 
L'existence de groupes cosmopolites (G4, G5, G6) et d'écotypes dispersés non classifiés dénote de la riche diversité génétique au sein de la collection et laisse supposer l'existence de cotonniers issus d'introgressions naturelles entre cotonniers diploïdes (G. herbaceum et G. arboreum) d'une part, et tétraploïdes ( $G$. hirsutum et $G$. barbadense) d'autre part, à vérifier.

\section{Conclusion}

Les connaissances générales accumulées révèlent une riche diversité au sein de la collection.

Certains des écotypes ont subi des améliorations (G. hirsutum) tandis que d'autres sont restés presque primitifs notamment les écotypes à tendances pérenne et végétative. Des phénotypes rencontrés dans notre collection, notamment les fleurs à pétales jaunes sur des plantes de G.arboreum et $G$. barbadense, la fibre de couleur kaki sur des individus de G. hirsutum (pérenne) et des graines libres sur des individus classés comme appartenant à l'espèce $G$. barbadense n'avaient pas été signalés ni pour les cotonniers traditionnels du Cameroun (Seignobos et Schwendiman, 1991), ni pour ceux du Sénégal et de la Gambie (Bèye, 1989). Il ressort de cette étude que cette collection nationale est riche de 4 types morphologiques principaux :

- le type 1 comprend les écotypes majoritaires dans la collection, de port allant du type « gobelet » à «pyramidal » avec des feuilles palmées, des fleurs à pétales et pollen crèmes, à macule rouge absente, à fibre blanche, brune et kaki. Pour les paramètres de production, ils ont des valeurs moyennes (écotypes de G1) à bonnes (écotypes de G5). Pour les paramètres architecturaux, ils présentent des valeurs très faibles (G1) à faibles (G5). Ils seraient des écotypes proches des G. hirsutum, plus ou moins améliorés ;
- le type 2 est proche du type 1 au plan qualitatif. Il s'en distingue par la corrélation moyenne à forte de ses individus avec les variables architecturales. Une de ses caractéristiques principales est la production tardive à très tardive de capsules. Il s'agit de la majorité des accessions de G6 ;

- le type 3 pour lequel les individus se caractérisent surtout par des feuilles digitées, des capsules pyramidales et un duvet de couleur verte. Les pétales sont généralement rouges et rarement jaunes. Ces accessions sont majoritairement de port élancé, de taille grande à très grande, produisant un nombre impressionnant de BV et de sites sur BV. Cependant, ils produisent très peu de BF et de capsules sur $\mathrm{BF}$, du moins en première année de culture, pour la majorité. Ce type comprend des accessions plus ou moins précoces de $G$. arboreum et de $G$. herbaceum;

- le type 4 ( $G$. barbadense) est caractérisé préférentiellement par des feuilles palmées-à digitées et larges, des capsules coniques s'ouvrant très tardivement. Les graines sans duvet (nues) sont le plus souvent soudées en rognon et rarement libres. Au champ, les plantes sont de tailles courtes à moyennes, les squares évoluent difficilement en fruit. C'est un type tardif ou photopériodique.

Dans un contexte de changement climatique, cette diversité s'avérera très utile pour enrichir le pool génétique utilisable pour l'amélioration du cotonnier. Il serait impératif d'affiner la caractérisation et de vérifier d'éventuelles introgressions naturelles inter-espèces, de poursuivre les évaluations aux plans agronomiques et moléculaires (en rapport avec la technologie de la fibre, la résistance à certains stresses biotiques et abiotiques) telles qu'elles pourront intéresser les sélectionneurs du cotonnier. En attendant, il est urgent de prendre les dispositions appropriées pour sauvegarder cette riche collection aussi bien 
ex-situ que chez les détenteurs originels. La menace d'érosion génétique de ces cotonniers est bien réelle au regard du nombre très réduit des détenteurs actuels et de la faible valorisation dont ils font l'objet aujourd'hui.

\section{REMERCIEMENTS}

Les auteurs sont très reconnaissants au Projet d'Appui à la Filière Coton Textile (Paficot)-Burkina Faso qui a financé les missions de prospection-collectes ainsi que les essais d'évaluation des écotypes collectés au champ, en station de recherche.

\section{REFERENCES}

Bado BV. 2002. Rôle des légumineuses sur la fertilité des sols ferrugineux tropicaux des zones guinéenne et soudanienne du Burkina Faso. Thèse de Doctorat, Université de Laval, p. 197.

Bednarz CW, Nichols RL. 2005. Phenological and Morphological Components of Cotton Crop Maturity. Crop Sci., 45: 1497-1503.

Bèye AM. 1989. Etude des cotonniers subspontanés du Sénégal et de la Gambie : Identification de quatre types de cotonniers, UNIVAL, ISRA. 18 p.

Dessauw D, Hau B. 2004. Manuel de Description du Cotonnier (Gossypium spp.) $\quad\left(1^{\text {ere }}\right.$ edn $)$ Cirad Editeur: Montpellier, France ; 70.

Dessauw D, Hau B. 2006. Inventory and history of the CIRAD cotton (Gossypium spp.) germplasm collection. Plant Genet. Resour. Newsl., 148 : 1-7.

FAO. 2010. Deuxième rapport sur l'état des ressources phytogénétiques pour l'alimentation et l'agriculture. FAO, Rome, 412 p.

Fryxell PA. 1992. A revised taxonomic interpretation of Gossypium L. (Malvaceae). Rheedea, 2: 108-165.
Gotemare V, Singh P. 2004. Use of wild species for cotton improvement in India. ICAC Rec., 22: 12-14.

Hau B. 2000. Variabilité disponible pour l'amélioration génétique du cotonnier. In: Actes des Journées Coton du CIRADCA, CIRAD-CA (ed.), Montpellier, France, Programme coton du CIRADCA, 287-291.

Igué J. 2003. Le secteur informel en Afrique de l'Ouest : Le cas du tissu traditionnel. In L'Afrique de l'Ouest dans la Compétition Mondiale. Quels Atouts Possibles? Damon J, Igue JO (eds). CSAO-OCDE: Karthala.

Ibpgr. 1985. Cotton Descriptors (revised). International Board for Plant Genetic Resources: Rome; 20 p.

Lagandre D. 2005. Le secteur cotonnier en zone franc, entre succès et dépendance. AFD, Rapport thématique Jumbo, septembre 2005, Paris.

Levrat R. 2008. Le Coton en Afrique Occidentale et Centrale avant 1950 : Un Exemple de la Politique Coloniale de la France. Editions l'Harmattan: Paris ; 365 p.

Mergeai G. 2006. Introgressions interspécifiques chez le cotonnier. Cahiers Agricultures, 15: 135-143.

N'Doye O, Roy-Macauly H, Faye MD, Sangare A, Sérémé P. 2011. La recherche cotonnière en Afrique de l'Ouest et du Centre. In Glocal, revue africaine sur le commerce et le développement, $\mathrm{N}^{\circ} 4$ mars-avril 2011, Numéro spécial sur le coton, 37-39.

Père M. 2004. Le Royaume Gan d'Obiré : Introduction à l'Histoire et à l'Anthropologie. Editions SEPIA : Burkina Faso.

Sarr D, Mergeai G. 2009. Élargissement de la base génétique de la principale espèce de cotonnier cultivé Gossypium hirsutum L. par la création et l'exploitation de 
lignées monosomiques d'addition. Biotechnol. Agron. Soc. Environ., 13(1): 187-201.

Seignobos C, Schwendiman J. 1991. Les cotonniers traditionnels du Cameroun. Cot. Fib. Trop., 46(4): 309-322.

Sêkloka E. 2006. Amélioration de l'efficacité de la sélection pour le rendement en coton graine du cotonnier Gossypium hirsutum L. dans un contexte de nouveaux itinéraires techniques. Thèse de Doctorat, ENSA/Rennes, France, p. 192.

Singh VV, Punit M, Kulkarni VN, Baitule SJ, Pathak BR. 2003. Exploration within India for collection of cotton species germplasm. Plant Genet. Resour. Newsl., 136: 40-46.

Ulloa M, Stewart JM, Garcia-C EA, GodoyAS, Gay-tan-M A, Acosta NS. 2006. Cotton genetic resource in the western states of Mexico: In situ conservation status and germplasm collection for ex situ preservation. Genet. Resour. Crop Evol., 53: 653-668.

Upov. 2001. Guidelines for the Conduct of Tests for Distinctness, Uniformity and Stability. UPOV: Geneva 20, Switzerland; $26 \mathrm{p}$.

Valicek P. 1978. Wild and cultivated cottons. Cot. Fib. Trop., XXXIII(3): 363-387.

Wallace TP, Bowman DT, Campbell BT, Chee P, Gutierrez OA, Kohel RJ, McCarty J, Myers G, Percy R, Robinson F, Smith W, Stelly DM, Stewart JM, Thaxton P, Ulloa M, Weaver DB. 2009. Status of the USA cotton germplasm collection and crop vulnerability. Genet. Resour. Crop Evol., 56: 507-532.

Zoundi JS, Hussein K, Hitimana L. 2006. Libéralisation de la filière coton et innovation agricole en Afrique de l'Ouest. Cahiers Agricultures, 15(1): 1721. 